\title{
Reports and Surveys
}

\section{ADVANCED CYBORG (BIOLOGICAL ROBOT)}

Whilst research continues to produce robots that mimic nature's creatures, scientists at the Northwestern University in Chicago, USA have developed a robot that is controlled by the brain of a sea lamprey. This is therefore an example of a biological robot, perhaps the first of many that will be developed.

The robot is described as a creature which has a mechanical body fitted with wheels, motors, circuit boards and light sensors but is controlled by the brain of a sea lamprey.

Despite having only a few nerve cells from the sea lamprey it has already learned to follow or avoid lights. This has given encouragement to those who believe that biological robots have an important future and may become part of a new technology that would see artificial limbs or other body parts connected to the user's brain.

The research team at Northwestern University was led by Dr Ferdinando Mussa-Ivaldi. The team was able to remove the brain stem and part of the spinal cord from a primitive salt water fish under general anaesthetic, keeping it alive in a cold salty solution. A report in the New Scientist published early on in the researches said that:

"the team isolated a group of large nerve cells called Muller cells. These help lampreys to orientate themselves in water. Electrodes attached to the neurons allowed them to be stimulated with frequencies they would normally receive in the fish's body. When lights were flashed at the robot, the lamprey brain cells learned how to control the motors. The cyborg was able to follow and dodge a moving light source and move in a circle."

Developments by the team since this report indicate their confidence in their ability to apply their researches to medical applications. In particular, they suggest that they may well be able to provide the advanced techniques which would enable better prosthetic limbs for disabled people to be built.

\section{ARTIFICIAL VISION}

The search for a high-tech, method for producing artificial eyesight is now reaching its climax with the claim from Japan that their scientists will be able to restore vision to millions of blind people within the next few years.

Three universities are collaborating with the Japanese government in research which is supported by $£ 15 \mathrm{~m}$ of State money with the goal of putting 'artificial eyesight systems' on the market by the year 2005. A report from Tokyo says that the Japanese scientists are developing:

\begin{abstract}
"new photodiodes - semiconductors that can turn light into electrical signals. These are intended to simulate the retina, the sensitive layer at the back of the eyeball. The retina sends impulses via the optic nerve to the brain where a visual image is formed."
\end{abstract}

Research into artificial eyesight is not confined to Japan but is a current research area worldwide. In the United States experiments have already been conducted with silicon chip implants that were expected to restore some perception of light and shade. Initial results were said to be disappointing because the signals generated were not strong enough. The Japanese, however, are more optimistic and say that:

"The signs so far are promising, according to our specialists in this technology. We think we can give people more than just light and shade perception. Japanese society is one of the fastest ageing populations in the world and one of the future problems we are facing is declining eyesight. This has large human and social costs and we want to be able to give people good eyesight that will last a very long time."

Researchers in Osaka, Nagoya and Nara universities are developing two methods to help people regain their sight. They are:

(i) A tiny but high-powered photodiode is implanted in the retina.

(ii) A light-sensitive device is attached to a pair of glasses and effectively records an image. This image is transmitted in the form of infrared rays to another device embedded in the retina.

Researchers at Nagoya University say that they do not expect a fine image at the beginning of the development but colour vision will be the next step and, in the long term, they believe that completely normal eyesight will be achieved.

Research projects such as the Japanese Government's collaboration with the universities is very encouraging. Public money has been pledged to support the venture because of the social and human costs of an ageing society. Other countries, it is hoped, will also appreciate the need for such endeavours. Many researchers in this field have also seen the possibilities of transferring this technology to many other projects. Turning light into electrical signals that can be read by the brain whether human, animal, part biological, or just artificial, efficiently, has many other potential applications. Biological robots may require to see and signal links to a 'brain' will be essential. Neuro-robots will also need visual systems which will involve connections to neuro-nets or whatever hardware is finally conceived. The 
fact that artificial eyesight for humans may soon be a reality will in itself more than justify the enormous efforts now being made worldwide to achieve it.

\section{BIOMETRIC TECHNOLOGY}

\section{III.1. Background to biometric technology}

Over the last decades Biometric Technology has provided the information technology (IT) field with a number of innovative systems. Many have been solely concerned with system security and a number have been discussed and reported upon in these pages.

Biometrics is concerned with the use of machines to recognise humans by means of their physical or behavioural features. It soon became apparent that system security was becoming one of the major concerns of IT providers to business and industry and indeed to anyone who wished a secure environment or 'system'. System here can mean anything from a credit card company's facilities to a computer used on any application or operation. The result has been an enormous demand for efficient and usable security. The technology has to embrace a wide range of practical uses that range from protecting sensitive information to the physical control of welfare payments.

Examples of the range of these applications were given in Biometric Technology Today which has reported the use of face and fingerprint recognition systems to weed out troublemakers at Netherlands night clubs; iris patterns were used for security at the Olympic games in Australia; and Korean telephone manufacturers are using fingerprint technology to enable secure e-commerce via mobile phones. In addition, other emerging areas involve providing secure access to company intranets for remote staff needing access to sensitive information and in e-commerce, where consumers need systems they can trust.

In an article in The Computer Bulletin (September, 2000, pp. 20-21), Mark Locki asks the question: Why is this technology coming to the fore? He suggests that currently there are too many security layers. He gives the example of the three-level security system which starts at level 1 with a PIN number, at level 2, a plastic card or a key and at level 3 , something more sophisticated.

All are to be linked to the person who wishes access, maybe something to be known, something you have, something you are or a combination of them. We are all familiar with the deficiencies of these security levels. It is to biometrics that we now turn for a system that may give 'absolute verification' of a system user. It offers the highest security level because it relies on our unique physical or behavioural characteristics.

\section{III.2. Some current developments}

The development of smartcards appeard to solve many of the problems of security but, of course, there is no guarantee that the person using the card is indeed the authorised user. Like the ISO standard card, now some 40 years old, it is not impossible to copy or change even the most sophisticated card. Biometrics therefore offers the opportunity of using some unique physical or behavioural characteristic of the human, and one that can be checked automatically. Since such characteristics can vary, biometric systems are designed to allow the accuracy to vary, often in relation to the level of security required. That means that in an office involved with financial matters, 95\% accuracy will suffice but in a defence establishment some $99 \%$ may be a requirement. Some of the physical attributes at present used or being developed include:

(i) Fingerprint - already used extensively.

(ii) Facial characteristics - computers can now recognise faces.

Using the eye - the pattern of veins at the back of the eye and the iris are currently used.

(iii) Hand - the shape of the bones in the hand have been found to be unique.

(iv) Veins - patterns of the veins, particularly at the back of the hand are also used.

Other physical characteristics are also being investigated. It would seem, however, that behavioural biometrics may also prove fruitful. Behavioural biometrics allows identity to be checked using dynamic processes. Traditionally this has been the person's signature, but now also includes the way we type. Signature verification was one of the first projects for many of the first computer scientists who regarded it as 'something of a challenge'. Some fine algorithms have been constructed for this purpose, many of the current ones taking into account variation in time so that the template for a behavioural reference has a changing template for reference.

\section{III.3 Requirements listed for investigation}

Mark Locki, who is the editor of Biometric Technology Today, lists at least five requirements that should be investigated to find out how a particular biometric suits a particular application, they are:

(i) Public acceptability: accepted by those who will use it.

(ii) Work and Cleanliness, age, diseases they may environmental suffer from, their profession and factors-users: even gender can affect performance.

(iii) Ease of use: This is extremely important in consumer applications because if customers find a biometric device intrusive or difficult to use they will take their custom elsewhere.

(iv) Verification This examines the biometric performance: system's accuracy in comparing the latest reading with the user's template.

(v) Template One biometric system needs only Memory size: nine bytes of data, which could easily be stored on a magnetic stripe card; others need the storage potential of a smartcard or a computer.

One other debate currently in progress is how the performance of these systems should be assessed. What, for example, is the 'error rate' of the system? How robust is it? 
How does the system integrate with other systems? and many other questions are asked of the manufacturers of such systems.

\section{III.4 Future initiatives}

We are told that the development of a general biometrics applications programming interface (API) was required if such systems were to be adopted. In 2000 an industry-wide BioAPI was released. Unfortunately, matters are not as easily resolved in the software world and the situation became very confused when some companies went their own way. We are told, however, that all is not lost and "the prospect of having a uniform operating system and integrated biometric API still seem possible". We are also told that the systems are 'very affordable' with the average price per access point being just under $\$ 500$ (£310) in 1999 compared with $\$ 6,000(£ 3,700)$ six years ago!

No one can doubt the importance of biometrics technology and its place in the future of the more advanced security systems. In particular, those involved in research and development will see the newly implemented systems as of special relevance to automation.

We are, of course, reporting on systems that are being used in the real world and about new initiatives such as the development of cash machines that use iris recognition for identifications, as well as many other pioneering initiatives using biometric technology.

\section{BIOMIMETICS}

\section{IV.1 New branch of science}

One of the newest branches of science that is of direct interest to readers working in the fields of automation and robotics is called biomimetics. This is the study which aims to create synthetic copies of organic matter. In particular, researchers in this area have studied animals and insects that are capable of surviving in extreme environments. They are investigated to see how they could be adapted so that their qualities and skills can be imitated in machines. The researchers aim to be able to copy these natural structures to build robotic systems. There have already been numerous examples, worldwide, of such creations. One such research centre is the Office of Naval Research (ORN) in Virginia, USA. In addition the US Pentagon's Defence Advanced Research Projects Agency has been active in co-ordinating work designed to develop the building of robots that copy natural structures.

\section{IV.2 Progress of researches}

The Pentagon, we are told, has been supporting such innovations for over ten years with the result that many of the prototype robots are regarded as being only a few years away from active service. The Projects Agency is reported to be spending more than $£ 6 \mathrm{~m}$ a year on these initiatives with many reaching their preliminary field tests. Some of the machines on the list of projects have already been well publicised in these reports. Currently, on the drawing board are robotuna, roboscorpion and robofly. All have potential roles to play in both military and civil scenarios. When a UK newspaper recently published details of these devices on the 1st April, it was accused of an 'April Fools' spoof. In reality, however, scientists are deadly serious in their pursuit of any adaptation of animal or insect studies to their design strategies.

\section{IV.3 Application to military operations}

One of the devices that has received publicity recently was the electronic robolobster. Unfortunately, full scientific details of the device has not been reported in the literature, but ORN researchers have reported that they are developing a device which will be about 18 ins. long and modelled on a real crustacean. One of its uses would be to help the military in frontline warfare. It could, we are told, be used to detect mines ahead of troops mounting operations from the sea. A brief description of the device says that it is:

"powered by a battery and it copies the way a lobster adapts to irregular seabeds, manoeuvres at different depths and copes with tumbling surf and changing currents. Signals from antennae and hair sensors are processed in its computerised brain and used to control its bionic muscles. The robolobster would survey an area and report back that it had found an object and either destroy it through a self-destructive process or allow troops to be made aware of the danger."

\section{IV.4 Other projects}

Whilst the robolobster is a likely candidate for further development, other more bizarre devices that mimic nature's creatures are being researched, particularly at universities and institutes in the United States. Many of these projects aim to produce high-tech products modelled on insects, crustaceans and marine wildlife. All have the support and encouragement of the US government's defence agencies which already see the wide range of potential applications for such systems. The possible 'spin-off' to non-military uses is also not being ignored.

\section{EFFECT OF TECHNOLOGY ON THE MIND}

A report from Japan suggests that memory dysfunction among the young requires immediate investigation. Dr Toshiyyuki Sawaguchi, who is professor or neurobiology at Hokkaido University's School of Medicine said that:

"They are losing the ability to remember new things, to pull out old data or to distinguish between important and unimportant information. It is a type of brain dysfunction. Young people today are becoming stupid."

In the United Kingdom we are told that whilst there have been no formal studies there is an increasing belief amongst many psychologists that a growing number of people in their twenties and thirties are suffering from severe memory loss because of increasing reliance on computer technology. In a recent interview with a UK National Newspaper, Professor Pam Briggs of Northumbria University (UK), who was the chair of the British Psychological Society symposium on the effects of technology said that: 
"I think increased use of the internet and computer technology is starting to have an effect. Everyday memory might be at threat if you are using the computer as a kind of external memory."

Whilst no researches specifically directed at the problem are available, there is no doubt that many institutions will take up the concern, displayed by many scientists and begin formal researches. Meanwhile, there are numerous reports and anecdotal evidence about the problems encountered. Some of the quoted cases cited, for example by Dr Takashi Tsukiyama, who has a private clinic in Tokyo, support the present preliminary reports. He says that he has seen an increase in severe memory problems and in the past two years reports that more people in their twenties and thirties have presented themselves with memory impairment. Another opinion on these effects on memory comes from Dr David Cantor of the Psychological Services Institute in Atlanta, Georgia, USA who is quoted as saying that:

"Many experts believe information overload is making it difficult for some people to absorb new information, as they have reached the limit of what they can store in their brains. These people forget things because they were too distracted to absorb them in the first place."

Dr Cantor has treated patients for memory and attention loss for more than twenty years.

On the basis of the current evidence there seems to be no doubt that sufferers in this young age-group are increasingly complaining that they can no longer recall names, written words, appointments, and in some cases quoted have had to give up their employment. The blame, say many doctors, rests with the ever growing numbers of people who use computer technology, electronic organisers and such aids as automatic car navigation systems. These devices, it is claimed, lead to diminished use of the brain to work out problems and inflict 'information overload' that makes it difficult to distinguish between important and unimportant facts. One preliminary study in Japan suggests that of 150 people aged between 20 and 35 more than one in 10 are suffering from severe problems with memory.

Unfortunately, scientists themselves are their own worst enemies in that more and more high-tech gadgets are being produced with such easy-to-use interfaces that they provide readily accessible add-on power to the human brain. For example, the first computers of the 1950s era required their users to think and to construct quite complex structures in programming languages. Now interfaces which access great banks of memory and vast arrays of software, all functioning at high speeds, present fewer challenges to the brain. The brains of the young who are more readily exposed to this new way of both living and working may well be adapting themselves to life in a high-tech world.

\section{IMPROVED IMAGING ALGORITHM (APPLIED TO GUIDING AN UNDERWATER REMOTELY OPERATED VEHICLE (ROV))}

The problems of interpreting images under conditions of extremely poor visibility are being ivestigated by SIRA and University College London (UK). As part of a joint
Postgraduate Training Programme Faraday research project, researchers have developed a new algorithm to assist in the interpretation of poor visibility images.

When dealing with images of very poor visibility, it is essential to use all of the limited amount of information available, such as prior knowledge of the scene. Knowledge of the approximate position of the camera can also be useful. For example, if you have an idea of the position of the camera, you can estimate what you expect to see if you have a model of an important object in the scene. This position can then be refined based on the images received. The process involves first distinguishing the object from the background, and secondly matching a model of the object to the segmented image.

Previous research has looked at matching a model directly to the image data and a variety of strategies have been proposed to solve it. However, many of these solutions require high quality images and a good initial estimate of where you are and what you are looking at. Much less attention has been paid to real world conditions, such as those encountered when guiding an underwater remotely operated vehicle (ROV).

The project is being carried out by Rustam Stolkin, a SIRA/UCL* associate, together with Dr Mark Hodgetts (SIRA Ltd) and Dr Alistair Greig (UCL). It is one of the research programmes in instrumentation in the Postgraduate Training Programme operated by SIRA and UCL, which is sponsored by the Department of Trade and Industry. This team has developed an algorithm which estimates the current camera position based on knowledge of the vehicle's recent motion, using a predictive filter. A predicted (and segmented) image is then generated by projecting a 3-D model of the environment onto an image plane at the estimated position.

This predicted image is used to interpret the observed image using a technique known as Extended-Markov Random Field (E-MRF) segmentation by combining what the system sees with what it roughly expects to see. The resulting segmented image is then compared with the environment model to provide a new estimate of the camera position. This improved position estimate can then be fed back into the start of the algorithm, to produce progressively better estimates.

Essentially, this means that knowing roughly where you are improves what you can see, which in turn improves your knowledge of where you are. This creates a positive feedback loop, resulting in more accurate interpretation of the image.

As an example, the algorithm was applied to the problem of underwater navigation of an ROV. These unmanned submarine vehicles are used in the inspection of offshore oil-rig structures. The images used show a scale model of a typical offshore structure, fabricated from welded steel tubing. The images were captured by a "roving eyeball"

\footnotetext{
* SIRA is a leading UK independent Research and Technology Organisation in the field of intelligent instrumentation, and has expertise spanning sensors, image acquisition, sensory information processing, artificial intelligence, data mining and data visualisation. Sira contact: e-mail: diane.mcgraw@sira.co.uk
} 


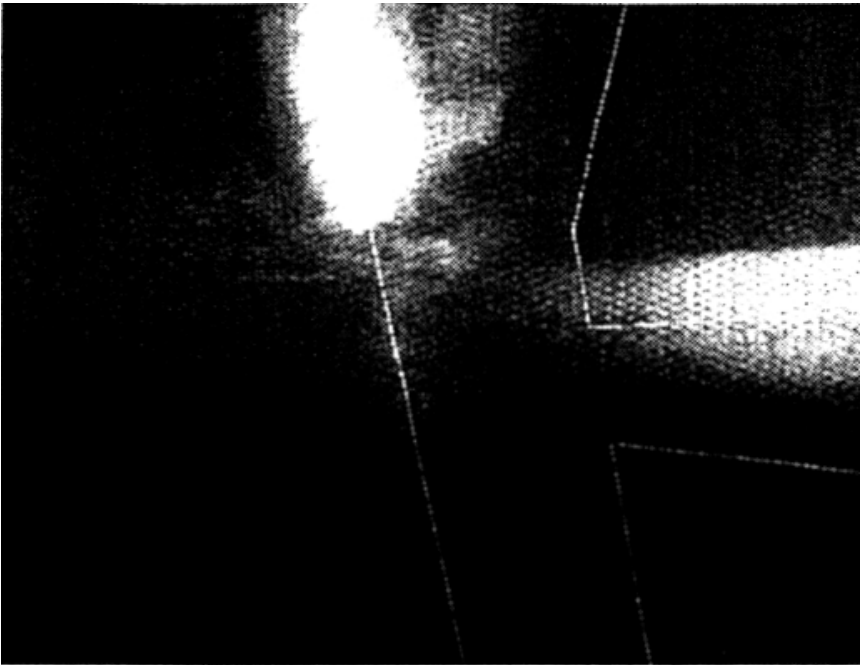

Fig. 1. Observed image of a typical underwater structure, overlaid with an initial prediction.

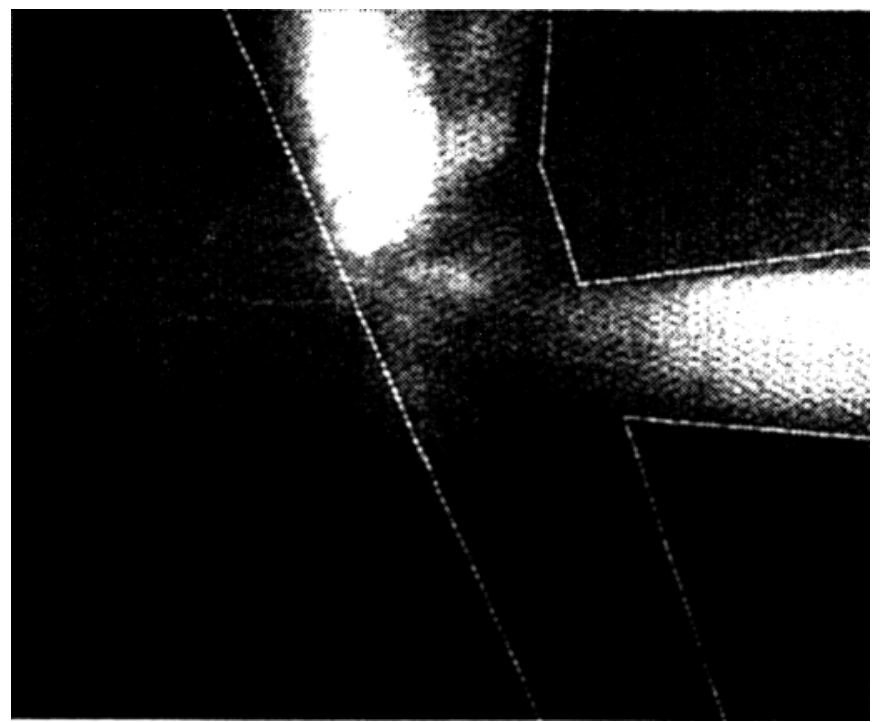

Fig. 2. Final estimate of predicted image.

mini-ROV at night. The only illumination was from lights mounted on the ROV itself.

The initial estimate of the camera position was generated from the results of the three previous images using a simple second-order predictive filter to model the motion of the ROV. During successive iterations, the algorithm reestimated the probability distributions for object and background. The algorithm progressively learned to distinguish between the object and the background by learning that "background" was consistently dark whereas "object" was brighter and had a greater intensity variation.

The algorithm can be seen to segment successfully a very poor visibility underwater image (Fig. 1) and significantly improve an erroneous initial estimate of camera position and orientation relative to the structure in the image (Figs. 2 and 3). The results demonstrate a clear improvement over both conventional and model-based scene interpretation (These figures were part of a report release by the Company sent to the Editor on January 10, 2001).

Making use of prior knowledge enables the algorithm to interpret parts of the image that contain little or no useful

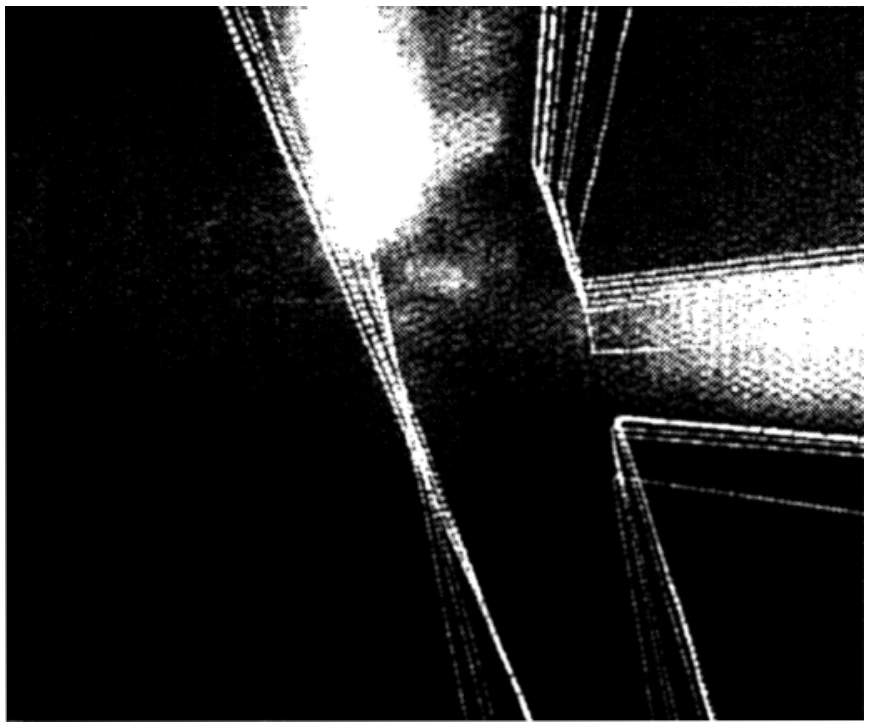

Fig. 3. Final estimate showing successive iterations.

information, producing interpretations similar to those arrived at intuitively by human observers. The algorithm learns about its environment with each successive iteration and adjusts the relative contributions of the predicted and observed information by responding to the visibility conditions at any given moment and in any given portion of the image.

The three-year project is scheduled to end in mid 2002. Applications are foreseen in the offshore industry, and any environment with very poor visibility.

\section{INNOVATIVE PRODUCTS AND PROJECTS}

\section{VII.1. Microchips made of plastic}

Scientists in the United Kingdom report that they have developed ultra-cheap plastic microchips that can be printed almost as easily as a colour magazine.This opens up the possibility of bringing 'intelligence' to everyday objects. It means, for example, that the new chips can be printed on clothes, paper, food containers and so on, making it possible for the user to be able to receive information about the item used.

The developers have set up a company Plastic Logic to continue the initiative. The company was set up by Professor Richard Friend who is the Cavendish professor of Physics at the UKs Cambridge University, and his colleague Dr Henning Sirringhaus. The company has developed and patented the technology.

The chips are to be made by spraying tiny globules of conducting plastics on to any material. The aim of the development, Professor Friend says, is to be able to transfer low-level intelligence to everyday products. Scientists have seen plastic chips as a much desired technology and have awaited its development for some time.

It has been said that such chips will in fact 'revolutionise' semiconductors. It is, however, accepted that they will not be as powerful as silicon versions but the obvious advantages are apparent. They will be flexible, very thin, we 
are told, and many thousand times cheaper to manufacture than current chips.

It is anticpated by the developers that the technology will be available in a usable state in some five years time. This announcement at Cambridge University will, of course, encourage other research institutions to compete with what is now a patented technology.

\section{VII.2. United Kingdom Research Programme}

The United Kingdom research initiatives into electronic devices and circuits based on silicon and germanium ( $\mathrm{SiGe}$ ) has received further encouragement from the nation's Engineering and Physical Sciences Research Council (EPSRC). This follows on from the successful first phase of its 'SiGe Initiative'. Some $£ 5.6$ million has been awarded to the two major groups involved with the programme so that they can continue with their research. Over $£ 11$ million has, to date, been invested into research on electronic devices and circuits based on SiGe. The programme of research was initiated in 1997 and since then $\mathrm{SiGe}$ has built into a successful activity involving industry and has attracted many forms of investment. It is reported that phase one of this activity is close to completion and has already made major improvements in semiconductor device performance.

It is reported that leading edge work has been carried out on SiGe bipolar devices including carbon in the structure, and that on $\mathrm{SiGe} \mathrm{CMOS}$, where P-channel performance equivalent to that of $\mathrm{N}$-channels has been published.

Professor Peter Saul of Saul Research, who is a consultant who has the task of focussing the initiative for the research community, says that:

"In high-speed digital circuits, on a given process geometry, you're probably going to be able to operate two to three times faster with this, compared with conventional technology."

The work has received great impetus from the Information Technology \& Computer Science (IT\&CS) and Materials which supported further research following a successful SiGe community workshop held in November 1999. A report of this meeting says that:

"The event gathered academics and industrialists interested in SiGe related to its growth, characterisation, device design and fabrication and provided an excellent opportunity to foster dialogue between academic researchers and users from industry.

It was possible to gauge the nature and scope of $\mathrm{SiGe}$ research worldwide and compare it with the UK. There were presentations from key industrialists and the participants benefited from hearing about current developments across a wide field of activity.

The conclusion drawn from this meeting was that a further phase of research would be timely, so to provide a process that would enable co-ordination between existing participants and to encourage new entrants, Professor Saul brokered discussions with potential academic researchers and commercial users to generate a well-balanced portfolio of projects."

The two phases of the work involves:
Phase I - includes two large groups - HMOS (High Performance metal-oxide semiconductor) and HBT (Heterojunction bipolar transistor). These groups came back with the projects for the following phase.

Phase II - which was subject to peer review, resulting in a recommendation of funding for both the projects.

As a result, the HMOS group has been awarded $£ 4.3$ million and the HBT group $£ 1.3$ million. The groups hold regular project meetings to make sure the research runs smoothly. Matters such as intellectual property (IP) are being discussed, as well as the means to protect it.

There appears to be little doubt that SiGe technologies, especially the BiCMOS form, will dominate future designs in the near to medium term. We are informed that, in particular, the technology makes new radio frequency circuits possible. Other results and applications will no doubt be forthcoming and contribute to this important area of research and development.

Further information about the programme is available from:

Professor Peter Saul: email: peter@saulresearch.co.uk EPSRC - Tel: (UK) 01793 444343; email: chris.nott@ epsrc.ac.uk

\section{VII.3. Carbon Nanofabrics Projects}

Researchers believe they have found a way to make the 'everlasting' fabric. Dr Ray Baughman, Honeywell International, New Jersey, USA, writing in Science (November 2000) describes the new work:

"Scientists are fascinated by nanotubes, filaments of pure carbon less than one ten-thousandth the width of a human hair which have unparalleled strength - higher than any other known material, and are "spectacularly damageresistant". But the problem was to find a way to assemble trillions of these nanotubes into long fibres that preserved these extraordinary properties."

It is reported that now Dr Brigitte Vigolo and her colleagues at the Universities of Bordeaux and Montpellier (France) have created the first spinning process to make fibres from nanotubes. The report says that:

"They assembled the fibres by dispersing nanotubes which look like soot - in a detergent solution, and injecting this solution into a flowing stream of polymer solution. The nanotubes condense out of the polymer flow, which aligns them into ribbon-like fibres."

A spokesperson for the research team says that basically our fibres are like hairs and they consist of dense assemblies of trillions of aligned nanotubes. The researchers say that:

"The fibres can be strongly bent without breaking, and even tied into knots. Long and ultra-strong nanotube fibres could be used as artificial muscles, wires and superstrong materials.

Although the fibres are not as strong as the individual nanotubes, we think it will be easy to make them realise their theoretical potential, up to 100 times stronger than iron and six times lighter." 
The French Team believe that by building on their discoveries, it will be possible to devise an economically viable process for spinning strong nanotube fibres. All sorts of applications will then follow. As to producing the 'everlasting' garment, this is, they claim, becoming closer to reality. At the present stage of development such a garment would be very costly since nanotubes now cost $£ 350$ per gram. Even so, the researchers believe that the potential applications of carbon nanofabrics are both promising and exciting.

\section{PC POWER HARNESSED}

When the power of hundreds of thousands of Personal Computers (PCs) is harnessed, problems that have remained insolvable for generations can be tackled. Already PCs have been harnessed for extraterrestrial applications where their combined power enables scientists to predict potential breakthroughs. We already know the effect of using a large number of PCs, with or without their owners permission, to target websites. Power in numbers is not a new concept. The aim now is, however, to direct this massive and untapped computer power for the good of our society. The plans of the UK's Oxford University's Department of Chemistry to mobilise PCs to search for a cure for leukaemia in their spare time are surely in this category.

Their project has already been called the largest computational chemistry project ever undertaken. Its basic aim is to tap into the unused power of idle computers around the world to identify compounds that could become tomorrow's drugs.

We all know that for most of the time the average computer is left unused with many switched on all day just to display a screensaver. With the co-operation of the US company United Devices and the chipmaker Intel, the Oxford researchers aim to capitalise on this wasted power. They cite the similar project carried out recently that enabled PC owners to join the search for extraterrestrial intelligence where volunteers downloaded a free Internet program and installed it on their system. This enabled their computer, whenever it had spare time to run the program. The Oxford system works in the same way and the program will screen millions of chemical compounds for their potential ability to interact with four proteins linked with leukaemia.

Commenting on the project to the London Times (April, 2001), the Director of the Centre for Drug Discovery, at Oxford University, Professor Graham Richards, said that:

"One in four of the people throughout the world contracts some form of cancer. People now have the opportunity to donate their unused computer power, which will enable us to accelerate our programme of research and come up with many new molecular candidates that could be developed into cancer drugs."

Computational chemistry is now very advanced particularly in its techniques for investigating properties of chemical molecules. In this case the techniques used involve the creation of three-dimensional models of chemical molecules and investigating how those molecules fit into the known clefts and crevices in three dimensional models of the target proteins.

Scientists regard this as a proven method for the design of drugs and one which has already been used successfully, for example, with the discovery of the flu drug Relenza in Australia. The Oxford research team, we are told, hope to achieve something similar with leukaemia using PC power, which incidentally was not harnessed in this way in the Australian project.

In the Oxford Project volunteer computer owners will download software called Think, which has been developed by Dr Keith Davis. They will be sent the structure of one of the four target proteins and 100 molecules to match it. If one of the computers finds a hit, it will automatically send the information to the Oxford Team the next time it is logged on to the Internet.

The scale of the operation can be seen when it is appreciated that the idea is to study hundreds of millions of molecules, a task we are told, that would take 24 million hours of computer time, that is nearly 3,000 years, if run on a single machine.

The collaborating US company United Devices have already predicted that:

"Depending on the results of this program, the time to develop a new treatment and drugs could be cut from 12 years to five. Internet distributed computing allows scientists to consider projects previously believed to be considered impossible."

There is no doubt that harnessing such distributed computing systems has enormous potential for the study of a multitude of different problems in many differing fields of endeavour. Both the extraterrestial and the molecular projects will provide valuable data on how to organise such studies. Not all studies, however, will attract the attention of PC owners in the way the leukaemia project has, and it has been speculated that incentives may soon have to be devised to encourage and motivate potential volunteer PC owners to cooperate.

\section{REPORTS WORLDWIDE}

\section{IX.1. Israel - First intelligent machine?}

Artificial Intelligence Enterprises (Ai), an Israeli company, claims that they have developed a computerised toddler, which they have named Hal, that developed the linguistic abilities of a child of 15 months. This achievement they believe makes it the first form of artificial intelligence to understand human language. The inventors believe that it is the first to satisfy a standard test of a true mechanical mind. Unfortunately, many 'high-tech' development claims are disputed by other researchers and developers working at other institutions and organisations. Even so Hal does appear to be a project that is well worth publicising. A report on Hal says that:

"The Hal software, which is compact enough to run on a laptop computer, learns in a similar fashion to children, using problem-solving rules called algorithms to make sense of the stimuli it receives from a human 'carer'. 
As the human carer types in children's stories and responds to Hal's behaviour with encouragement or admonishment, like a parent, the machine learns to interpret words and contexts. It is capable of speaking a few simple words, and may eventually develop the language capacity of a child aged five."

When the project was announced last year many $A I$ experts were sceptical of some of the claims made. Even so, all expressed interest and wished the developers success. The general opinion was that the software described would need to be more advanced in concept if Hal was to exhibit what they described as 'true intelligence and understanding'. The suggestion, for example, that Hal would pass the Turing Test was questioned. The test, which it will be recalled, holds that if people are unable to tell the difference between a conversation with a human being and a conversation with a computer, that computer could reasonably be described as intelligent. Even if there was evidence that Turing's test was satisfactory many researchers believe it to be a 'false measure'.

\section{IX.2. Russia-Neuro-computer}

A recent press report tells us that the world's first neurocomputer has been developed in Russia. Scientists in Moscow say that this is the "first artificial brain that is armed with the intellectual ability to compete with human beings". It is suggested that the device is so sophisticated that it could make complicated decisions and constantly evolve like a real biological entity. The Russian scientist is said to be Dr Vitali Valtsev, who has spent 30 years developing a 'truly thinking machine' which he believes will outperform all previous models. The new brain uses a model of the neurons in the human brain. It is reported that previous models had been based on neurons in the spinal cord, which were too mechanically similar to computers. The new machine is said to be far more sophisticated and able to make judgements according to circumstances and to learn from mistakes.

The current state of the development Dr Valtsev says, is that the artificial brain exists only as a set of computer commands rather than a tangible object.

Unfortunately, comments that it could 'ultimately rule over humans' and evolve into a 'tyrannical monster' do not help to give the project a measure of scientific respectability, however, possible they may be. Currently financial support is being sought and Dr Valtsev is said to be courted by many potential backers, including the Russian Army. We all, of course, remain very interested in such a project and await hard scientific details of the project. Many scientists are also developing similar style machines but there is a consensus that all are in very early stages of planning and design. We all await the first prototypes.

\section{IX.3. Sweden - Biotechnology uses for microrobots}

Dr Edwin Jager, Linkopings University, Sweden is the main author of a report that provides some fascinating information about microrobots and their future applications not only in the medical field but also in biotechnology.
There have been numerous reports about the use of microrobots in such applications as micro surgery. Now the Swedish scientists have developed tiny 'submersible microrobots that are barely three millimetres long and one millimetre wide. The applications already suggested include delicate microsurgery and as microscopic factory workers, to manipulate cells in desktop 'biofactories' for genetic analysis.

Since the report was published further possibilies have been highlighted including the use of these newly developed microrobots in blood, urine, brine or indeed any other liquid. This has raised the possibility that there may be many biotechnology uses for the devices. They could, it has been suggested, have miniature hands that would be able to pluck single cells, bacteria, multicellular organisms and other biological entities from a sample, and then transfer them to an analysis station.

The work continues and further progress reports are awaited.

\section{IX.4. United Kingdom - First 'conscious' machine}

Creating the world's first 'conscious' machine is a challenge gladly taken up by one of the UK's leading experts in AI systems, Steve Grand. His work has already been recognised in the UK by the award last year from the nation for his services to the computer industry. His current project is to build a robotic orangutang, and he has already built the arms, eyes and head of the robot. He is now engaged in connecting up its 'brain' with the hope that it will be completed this year.

The robot is named Lucy (robotic orangutang) and is specified to have fully functioning arms, legs and eyes but will not be specifically programmed to use them. Conventional robots usually follow set programs. It is intended that Lucy will learn how to move and react. Steve Grand said that:

"Lucy will start off with a brain which is pretty much a blank sheet, and she has to figure out for herself what happens when she tweaks particular neurons and what the light falling on her retina actually means."

The experiment is ongoing and if successful would surely be regarded as a great breakthrough and an enormous achievement. The developer in explaining the robot's design said that it would be preprogrammed with only the most basic 'motivation' such as the desire to survive and receive stimulation. We are told that everything else will have to be learnt using 'neural network' circuitry, which mimics real life by allowing programs to run spontaneously or at random rather than in a predetermined way. This is regarded by many scientists as an impossible challenge and the chances of success at this time are rated low, although many top scientists have recorded their faith in his endeavour.

It will be recalled that Steve Grand wrote the computer program Creatures in 1996. This created cyberpets which lived inside desktop computers and were capable of evolving and even expressing emotion. It is estimated that over a million copies of the program were sold. 


\section{SOFTWARE DEVELOPMENTS}

\section{X.1. Software for the visually impaired}

New software to help the visually impaired will soon be available to help them access the Internet. The innovative package will enable millions of visually impaired people to obtain the texts that accompany website information. Called WebTalk it picks out the main text on web sites and reads it back to the user. Unlike so many software products of an innovative nature this system is said to be available to users in the next six months.

Commenting on the new system, Bernard Richards, who is the Director of the UK's Hampshire Association for the Care of the Blind, said that:

"The windows setup on computers makes things extremely difficult for visually impaired people and web pages use lots of flashing and moving images which are lost on blind people. This is going to make a big difference. People who had previously had no computer experience or who have not got to grips with the Internet will now be able to use it."

Software developers are aware of the 'talking books' and other audio services that are available for the visually impaired and the new breakthrough to Internet services is a new and challenging advance. The production of computer interfaces for such users has always been a priority amongst researchers and now it seems we are beginning to open up a new world. There is no doubt that further developments in this area will provide greatly enhanced interactive 'talk systems' which will benefit all computer users.

\section{X.2. Software for embedded control systems}

MathWorks is the originator and author of what it describes as the world's leading application development language for engineers, scientists and technical professionals. The company,* say that:

"As an international organisation, we offer a wide range of tools for numeric computation, visualisation, modelling and simulation. The company's flagship product is $M A T L A B$, a high-level language for technical computation that has largely supplanted FORTRAN. Today the company has more than 500,000 users around the world, including scientists and engineers in academic institutions, commercial companies and government agencies. Founded in 1984, The MathWorks currently employs more than 650 people world-wide. Its headquarters are in Natick, Massachusetts, USA.

Originally established in 1984 as an independent company (Cambridge Control Ltd), the MathWorks' operation in Cambridge spearheads the British sales and support of MATLAB and the associated products. The

* Further details can be obtained from The MathWorks Ltd., Matrix House, Cowley Park, Cambridge CB4 OHH, UK. See also; http://www.mathworks.co.uk

Section editor's note: Other companies producing software for use in robotics and automation are invited to contribute to this dedicated software section. company's Cambridge Consultancy Group supports this and also provides independent world-class consultancy in control, digital signal processing, embedded software development and corporate application development. Typically, the Group works on automotive, aerospace, defence, medical, communications, digital signal processing, financial and telecommunications projects."

Currently the company is introducing what they describe as the world's most advanced model-based design software for embedded control systems development. Readers who have used design software or are interested in its contribution to robotics and automation development will appreciate the company's statement that:

"The new embedded systems design solution is based on The MathWorks' established range of design tools. A significant upgrade of its principal products has led to Simulink 4.0, Stateflow 4.0 and Real-Time Workshop 4.0 being introduced as part of the company's Release 12 . With these three major new products, control engineers and embedded systems designers can take an integrated, model-based approach to embedded systems design, rather than using the traditional method of writing specifications and coding software manually. They can develop complex embedded systems designs, starting with the initial concept and taking them through to full deployment on the production target. By adopting these tools, tests have demonstrated that weeks or even months can be eliminated from the overall process of designing embedded applications for the aerospace, automotive, telecommunications and industrial control industries. Users have experienced a significant increase in the efficiency and reliability of their systems.

The new versions of Simulink, Stateflow, Real-Time Workshop and their extensions provide an interactive, graphical modelling environment that allows users to design, analyse and accurately simulate models of complex, large-scale systems."

The new model-based design environment is, the company say, the result of usability tests with more than 500 customers. As a result, many significant enhancements and extensions were made to the MathWorks core products. In addition new functions have been added to support modelbased design, to increase usability, to accommodate large systems modelling and to ensure that automatically generated software code is reliable, accurate and efficient.

Finally, the company believe that a particular benefit of the MathWorks model-based design solution is that:

“... control engineers and embedded systems designers can spend more time investigating new design concepts and refining ideas rather than performing routine coding and debugging tasks. Since models are debugged before software is written, errors can be detected much earlier in the development process. Software code, documentation and test functions are translated directly from the model, so process errors are eliminated and productivity is increased." 


\section{SURGICAL ROBOTS}

\section{XI.1. A robot called Da Vinci}

There have been many reports over the years about the development of robots for specific medical applications. A number of such machines have been described in this section. A recent demonstration of a surgical robot called da Vinci showed the capabilities of such devices. The demonstration was arranged by Intuitive Surgical, a small US company of Mountain View, California. The company built the $\$ 1$ million robot, they say, to become an operating room fixture. It was displayed at the annual meeting of the American College of Surgeons held at the end of 2000. With no human help the robot, used scissors, scalpels, and forceps, cutting artificial tisses, threading a needle and tying neat stitches. However impressive this demonstration, the medical director of the manufacturing company was still not satisfied. He is quoted as saying that:

"It has got a long way to go - it's too big - it's too expensive - it doesn't have all the tools surgeons need."

Perfection is sought and he promises the company will eliminate these perceived drawbacks.

There is little doubt that surgical robots are going to be able to perform all sorts of medical procedures either with or without the assistance of a human surgeon. Whether it will operate alone is, ofcourse, another matter. Where it has been tried it has shown its potential. Its benefits to the patient are indeed impressive. They include: smaller incisions, less pain, fewer infections and other complications, shorter hospital stays, faster recovery, even improved odds of a successful outcome. The benefits to many health organisations is obvious with the possibility of cutting waiting times for operations and a more controllable financial budget.

The robot Da Vinci and similar machines are currently regarded as an extension to the surgeon with, in some cases articulated tools at the end of three arms inserted into the body through small openings. The tools need little room to manoeuver and the Da Vinci team of developers have given the robot the same manipulative skill, or degrees of freedom, as the human hand and wrist.

\section{XI.2. Current Applications}

A report in the U.S. News \& World Report (January, 2001) provides an update of past and current procedures carried out by Da Vinci. It says that:

"Da Vinci performed its first operation on a human being in March 1997. Doctors at the University of Dresden's Cardiovascular Institute have now performed many heart operations, including well over 100 beating-heart coronary by pass surgeries - a difficult procedure. Last July, the Food and Drug Administration cleared da Vinci for use in laparoscopic procedures, such as removing the gallbladder or surgically correcting severe heartburn through small incisions. The robot has been employed experimentally in Germany to remove cancerous prostate glands while sparing the nerves that control erection and the muscles that control urination."
Further details are also given in the report of $\mathrm{Da}$ Vinci's activities. In the United States, we are told, mitral valve repair has become Da Vinci's forte. This is the valve that regulates blood flow between the heart's left atrium and left ventricle. If the valve weakens or leaks it can cause heart failure and this condition causes thousands of deaths each year. In May 2000 at the East Carolina University's Brody School of Medicine in Greenville, N.C, U.S., a team with the aid of Da Vinci performed the first 'beating-heart mitral valve' repair in the country. The results were successful and the team have now completed more than a dozen successful valve repairs.

\section{XI.3. Future research and applications}

Many companies worldwide are engaged in producing surgical robots; many like Da Vinci are beginning to prove themselves in a variety of medical procedures. In the U.S., for example, robots for this application are also being produced by Computer Motion, Santa Barbara, California. In many medical centres the surgical robot has arrived and is slowly gaining a foothold. More sophisticated robots are being designed and manufactured based on the experiences gained in using devices like Da Vinci. Future generations of patients will undoubtedly benefit from these robotic initiatives.

\section{SMART BOMB SQUAD ROBOTS}

\section{XII.1. Problems encountered with the Andros bomb robot} At the United States Sandia National Laboratories, Albuquerque researchers are attemping to make bomb squad robots easier to use. Worldwide bomb threats by all sorts of terrorists and other organisations have meant that squads of trained personnel have to be on standby to defuse many different types of explosives. It soon became apparent that robots would be the ideal way of disarming bombs and many lives have been saved as a result. These robots are now much more sophisticated than the first devices that were seen on television trundling along roads towards booby-trapped vehicles. Even the current versions are, we are told, difficult to use and the National Laboratory at Albuquerque aims to devise software that will remove these difficulties. In 1998 the Police Department in Alberquerque spent $\$ 160,000$ for a remote controlled robot called Andros which had been built by the company Remotec, which is a subsidiary of Northrop Grummam. This robot was designed to pick up a suspicious device and put it in a safe container or use one of its tools to disarm or disrupt the bomb's mechanism. It was soon found, the Police Department officials say, that their officers were having difficulties in manipulating the robot. Although a very versatile device, Andros could climb stairs and manoeuver through difficult terrain, its controls proved hard to master, even when bombsquad officers trained with the device for some two hours each month. Its use there has proved limited, although it has been in action more than a dozen times. In some cases, however, officers had to complete the disarming themselves because they say they were not 'comfortable with their proficiency'. 


\section{XII.2. Building smarter software}

The design of the robot is not questioned and the makers have sold over 700 of them. A plan was put forward to adapt a piece of software developed at the Sandia National Laboratories for robots that would help clean up after environmental disasters. The software is called Smart, which stands for Sandia Modular Architecture for Robotics and Teleoperations. It is designed to use components, like sensors that are made by different vendors. As a result, instead of being manually controlled a robot equipped with Smart could be given sensors that would allow it to make some decisions of its own. The robot could work out the best path to a point in a room, center itself in a doorway or grip a bomb on its own. Thus leaving bomb squad personnel to focus on what steps to take.

A development programme of some three years is envisaged including testing and evaluation. It is predicted that a robot controlled by Smart software will be able to complete its tasks in half the time they do now. With Smart the robot could also be adapted to include any new threats posed by by bomb makers who are always challenging the bomb disposal personnel with new and more sophisticated explosive devices.

\section{VISION SYSTEMS}

\section{XIII.1. Computer vision systems}

Professor David Murray of the UK's Department of Engineering Science, at the University of Oxford has been exploring the use of zoom in automatic tracking. He explains that:

"Most of us will have watched TV broadcasts where skilled camera work enhances the information flow to the viewer. There seems every reason to think that a similar automatic capability would be of benefit to a computer vision system.

The movements of a camera and lens used to follow the action in, for example, a televised sporting event are typified by rotational and translational motions of the camera, and zooming of the lens. By comparison with the considerable attention lavished on automated motion tracking, automatic zoom control is a rather unexplored area."

The university's department is conducting research and exploring the use of zoom in automated tracking through its Active Vision Group. To an extent,we are told its work aims to:

"mimic human operator control, where camera zooming is initiated both purposefully and reactively. In the former case, some higher level process indicates that it would be valuable either to zoom in to collect more object detail, perhaps to help recognition, or to zoom out to obtain surrounding contextual detail. In the reactive case, the zoom setting is adjusted to preserve the image siz/e of the target as it moves away from or towards the camera."

Methods have been developed in the laboratory for automatic calibration for zoom-invariant tracking, for reactive zoom control and for Euclidean 3-D scene reconstruction from multiple zooming cameras. With these in place the researchers now aim to demonstrate these techniques in real-time surveillance applications.

Further information about this research and the project can be found on: www.robot.ox.ac.uk/ActiveVision/ Research/ZAP/ and also more general information at: www.robots.ox.ac.uk/Activevision/

\section{XIII.2. Echocardiography image processing}

A report on the development of 3-D echocardiograpic image processing methods by Dr Alison Noble of Oxford University UK (November 2000), Research File (Impact, No. 28,2000) outlines the methods and the background to the research initiative. She writes that:

"For many years cardiologists have used 2-D ultrasound imaging (echocardiography) to view the wall-motion of the left ventricle as a way to assess the healthiness of heart function. For instance, an ischaemic heart appears 'sluggish' with the ischaemic regions of the heart muscle exhibiting 'abnormal' motion. However, over the past 5 to 10 years we have seen a notable improvement in medical imaging technology, and a more prolific use of information technology in medicine. As a result, we can now image the heart in 3-D and cardiologists are beginning to demand automated quantification (numbers) rather than visualisations of the heart to help guide diagnosis and monitor treatment, and to remove some of the subjectiveness from clinical assessment."

This project concerns the development of 3-D echocardiographic image processing methods, particularly those that focus on sparse-view (or few view) reconstruction methods. The main difference from research on 2-D methods, we are told, is that 3-D echocardiography is a relatively new imaging method that has yet to establish its role in cardiac diagnosis and treatment planning and monitoring. It is emphasised that one of the key objectives of the research is to establish the foundations for clinical quantitative 3-D echocardiography.

At the Medical Vision Laboratory at Oxford University, Dr Noble leads a research team that has been focussing on the development of a fully automated method for quantitative 3-D echocardiographic image processing. A small clinical study on patients commenced in November 2000 at the Oxford John Radcliffe Hospital to compare the 3-D echocardiographic analysis methods being developed with nuclear medicine-based methods of clinical assessment. The research report tells us that:

"3-D echocardiographic analysis stretches current machine vision techniques to a limit. Ultrasound images are very noisy compared to conventional visual images meaning new approaches to image feature extraction (segmentation) are required, and the non-rigid deformation of a heart offers challenges in terms of tracking and motion interpretation. The Oxford group has developed some novel solutions to these problems.

A key factor limiting the acceptance of 3-D echocardiography in routine clinical practice is the relative 
poor image quality compared with 2-D echocardiography. Two-dimensional-array transducer technology, which provides 'real-time' 3-D echocardiography, is being developed as a solution to this problem by a number of the leading ultrasound physics groups around the world and should be available commercially in approximately three years time."

The researchers will, we are told, be working on the clinical validation of their methods with cardiologists at the Oxford John Radcliffe Hospital over the next two to three years. It is believed that as real-time 3-D echocardiography becomes commercially available they will be in a position to move their methodology into routine clinical use.

More information is available about this and other projects on: www.robots.ox.ac.uk/ mvl.

XIII.3. Developing a new 3-D imaging sensor

At the Heriot-Watt University, Scotland, U.K., members of the Departments of Physics and Computing and Electrical Engineering,in conjunction with Edinburgh Instruments and British Aerospace, have developed a new 3-D imaging sensor based on time correlated single photon counting (TCSPC).The researchers say that:

"Three-dimensional imaging is becoming very important for a wide range of applications. In the automotive industry it is used to create CAD models from clay prototypes, providing vital input to assess the performance and style of the final production model. In the aerospace industry, accurate 3-D data is required to check whether the aircraft meets tight specifications, and to act as input to packages which simulate aerodynamic performance

And 3-D images are captured to allow avatars to roam through virtual environments in the entertainment and leisure industries."

The Herriot-Watt 3-D imaging sensor has been used to create images from objects a few centimetres to several metres in working volume. It is claimed that it has achieved accuracies of the order of $15 \mu \mathrm{m}$ on single point measurement. It is reported that it is a 'time of flight' technique, but because it is responsive to single returned photons directly from the target surface, it has very accurate time, and hence distance, resolution. It is also explained that as it works at low levels of returned signal it is very sensitive and has been used to create 3-D images of distant, poorly reflecting and even transparant objects. The research teams in addition to the innovative hardware design have also developed a new approach to modelling and interpreting the received signal data.

Moves are ahead to find routes to industrial exploitation of the technique with new work continuing in a number of areas.

The report of the work in progress was authored by Professor Andrew Wallace of the Computing and Electrical Engineering Department of the Herriot-Watt University, who can be contacted on andy@cee.hw.ac.uk. More information is also available on:

www.cee.hw.ac.uk/Research/cvip_index.html

Professor B.H. Rudall

Norbert Wiener Institute and University of Wales (UK) 